\title{
Evaluation of Different Image Interpolation Algorithms
}

\author{
Ankit Prajapati \\ Computer Engg. \\ Parul Institute of Technology, \\ Vadodara, Gujarat.
}

\author{
Sapan Naik \\ Asst. Prof. in Computer \\ Science \\ Uka Tarsadiya University \\ Bardoli, Gujarat.
}

\author{
Sheetal Mehta \\ Asst. Prof. in Computer Engg. \\ Parul Institute of Technology, \\ Vadodara, Gujarat.
}

\begin{abstract}
Interpolation is the process of transferring image from one resolution to another without losing image quality. In Image processing field, image interpolation is very important function for doing zooming, enhancement of image, resizing any many more. Here in this paper, we have reviewed different interpolation algorithms. We have implemented all reviewed interpolation algorithms and done comparison of all.
\end{abstract}

\section{Keywords}

Interpolation, DWT, Filtering

\section{INTRODUCTION}

Approximating continues function's value using discrete samples is called interpolation [16]. Image interpolation is nowadays available in many image processing tools like Photoshop [1] and other so we thought the review of interpolation method will be useful for researchers. Most common interpolation techniques are nearest neighbor, bilinear and cubic convolution. Applications of image interpolation methods are image enlargement, image reduction, subpixel image registration, image decomposition and to correct spatial distortions and many more [13, 14]. In figure 1 we have shown the basic concept of how we can enlarge image using interpolation.

Digital image is a signal, spatially varying in two dimensions. This signal is sampled and quantized to get values. All these values called pixels of image. When we increase the resolution of image from low to high, it is called up-sampling or up-scaling while reverse is called down sampling or down scaling. In this paper we have presented algorithms for upsampling only. Two main categories are there for image interpolation algorithms called adaptive and non-adaptive. In non-adaptive method same procedure is applied on all pixels without considering image features while in adaptive methods, image quality and its features are considered before applying algorithm $[2,3]$.

In this paper we have studied many interpolation algorithms and implement all. We have done the comparisons of all these algorithms.

Paper organization is as follow. Section 2 contains non adaptive interpolation algorithms, section 3 contains adaptive algorithms, and in section 4 we have shown the comparisons

of all algorithms and finally section 5 contain conclusion and future work.
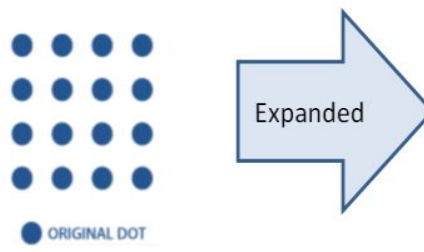

○ $0.0 \%$

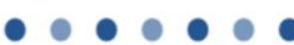

InTERPOLATED INEW Dor

Figure 1. Basic Interpolation Concept

\section{NON ADAPTIVE INTERPOLATION ALGORITHMS}

Without thinking or considering the content of image, we simply apply some computational in this methods. Normally commercial product like Adobe Photoshop cs5 uses this kind of interpolation methods.

\subsection{Nearest Neighbour Interpolation}

This method is very simple and requires less computation as it use nearest neighbor's pixel to fill interpolated point. This method is just copies available values, not interpolate values as it doesn't change values.

The interpolation kernel for each direction for this method is [16]:

$$
u(x)= \begin{cases}0 & |x|>0.5 \\ 1 & |x|<0.5\end{cases}
$$

Where $\mathrm{x}=$ distance between interpolated point and grid point

\subsection{Bilinear Interpolation}

In this method, interpolated point is filled with four closest pixel's weighted average. In this method we performed two linear interpolations, in horizontal direction and then linear 


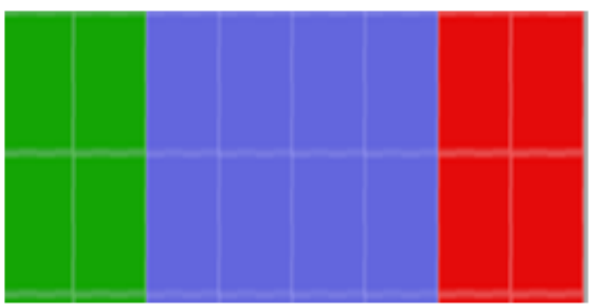

Original image

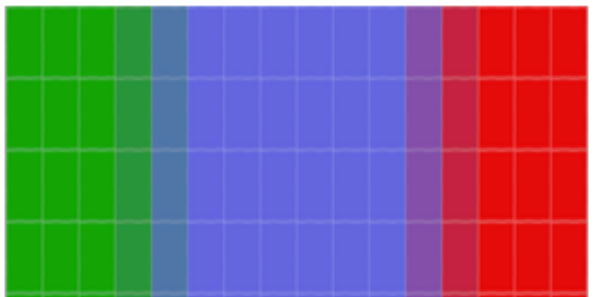

Bilinear

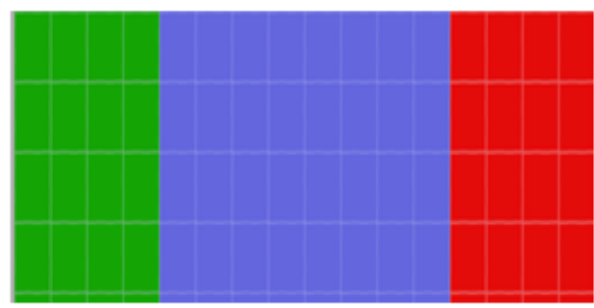

Nearest neighbor

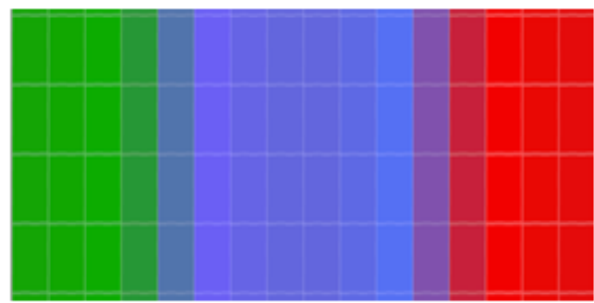

Bicubic

Figure 2 : Comparison of non adaptive methods

interpolation in vertical direction. We need to calculate four interpolation function for grid point in Bilinear Interpolation.

The interpolation kernel for linear interpolation is[16] :

$$
\begin{aligned}
& \mathrm{u}(\mathrm{x})= \begin{cases}0 & |\mathrm{x}|>1\end{cases} \\
& \{1-|\mathrm{x}| \quad|\mathrm{x}|<1
\end{aligned}
$$

Where $\mathrm{x}=$ distance between interpolated point and grid point.

\subsection{Bicubic Interpolation}

In this method, interpolated point is filled with sixteen closest pixel's weighted average. We can get sharper image than bilinear method.

The interpolation kernel for cubic interpolation is[16] :

$$
\mathrm{u}(\mathrm{x})= \begin{cases}\{3 / 2|\mathrm{x}| 3-5 / 2|\mathrm{x}| 2+1 & 0<=|\mathrm{x}|<1 \\ \{-1 / 2|\mathrm{x}| 3+5 / 2|\mathrm{x}| 2-4|\mathrm{x}|+2 & 1<=|\mathrm{x}|<2 \\ \{0 & 2<|\mathrm{x}|\end{cases}
$$

Where $\mathrm{x}=$ distance between interpolated point and grid point.

\subsection{Filtering-based(Re-sampling) Techniques}

Transforming image pixel from one coordinate to another(different resolution) is called Re sampling process. Digital image is discrete function in two dimension so we need 2D filtering or convolution. But it is computationally costly. We can separate 2D function and apply it first row wise and then column wise on 1D. We have studied different filters used for interpolation algorithm[1]. Filters are used for

\begin{tabular}{|c|c|}
\hline Filter & Reference \\
\hline $\begin{array}{l}\text { Michell cubic } \\
\text { polynomial filters }\end{array}$ & $\begin{array}{cc}\text { - } & \text { www.mentallandscape.co } \\
\text { m/Papers_siggraph88.pdf } \\
\text { - cognitrn.psych.indiana.ed } \\
\text { u/busey/erp/DigFilt.pdf }\end{array}$ \\
\hline $\begin{array}{l}\text { Bell quadratic } \\
\text { polynomial filter }\end{array}$ & $\begin{array}{l}\text { http://www.sweetwater.com/expert- } \\
\text { center/glossary/t--BellFilter }\end{array}$ \\
\hline $\begin{array}{l}\text { Hermite cubic } \\
\text { polynomial filter }\end{array}$ & $\begin{array}{cc}\text { - } & \text { http://en.wikipedia.org/wi } \\
\text { ki/Hermite_interpolation } \\
\text { - } \\
\text { http://www.bionixwallpap } \\
\text { er.com/help/faq/comparisi } \\
\text { on.html }\end{array}$ \\
\hline $\begin{array}{c}\text { Lanczos3 sinc-b ased } \\
\text { filter }\end{array}$ & $\begin{array}{l}\text { - en.wikipedia.org/wiki/La } \\
\text { nczos_resampling }\end{array}$ \\
\hline $\begin{array}{l}\text { Triangle Filter(linear } \\
\text { based) }\end{array}$ & 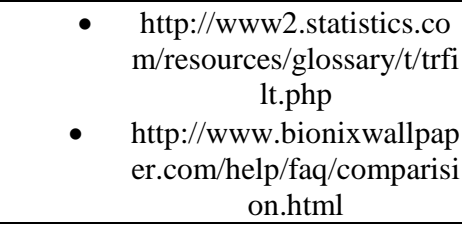 \\
\hline Cubic B-spline filter & $\begin{array}{c}\text { - } \quad \text { http://www.dannyruijters. } \\
\text { nl/cubicinterpolation/ } \\
\text { - } \quad \text { http://ieeexplore.ieee.org/ } \\
\text { xpl/login.jsp? D1163154 }\end{array}$ \\
\hline
\end{tabular}
blur and noise removal as well as edge identification. We have listed some of the filters and website from which one can learn more about these filters.
Table 1 : Filters and References

More about different filters can be found from http://lodev.org/cgtutor/filtering.html. Many research papers are also available for studying different filters.

\section{ADAPTIVE ALGORITHMS}

Adaptive algorithm basically uses some of the image features and improves interpolation result. Adaptive algorithm works based on different intensity present and local structure of image. Many adaptive interpolation algorithms have been proposed which enhance edges in images[7,10]. Local gradient information can also be used to enhance non adaptive interpolation algorithm. Normal problem with interpolation technique is blurring and blocking artifacts. This can be solved using directional interpolation. 


\subsection{Recent interpolation Algorithm}

We have studied some recent algorithms for interpolation. In [17], authors have presented edge-adaptive interpolation algorithm for Super-resolution reconstruction. Here they construct high resolution image using bilinear interpolation and detect edges. Then edges are refined using geometric duality between the low-resolution covariance and the highresolution covariance and local structure feature.

In research paper [18], authors shows new interpolation algorithm based on Pulse-Coupled Neural Networks (PCNN). Here clusters and the propagation paths of pulse are obtained by using the synchronous pulse burst property of PCNN. Different interpolation methods are used in the inner of clusters and at the intervals of clusters to complete all interpolations of image.

We have studied one research paper of Image Enhancement by Intensity Based Interpolation and Selective Threshold[19]. Here they have proposed one technique which determines edges by comparing the intensity variations between center pixel and its neighboring pixels of window size 55 . On the basis of designed thresholds, extrapolated blank pixels are filled.

We have studied one edge based interpolation algorithm presented in [20]. Here the gradient directions are explicitly estimated with a statistical-based approach. The local dominant gradient directions are obtained by using principal components analysis (PCA) on the four nearest gradients. The angles of the whole gradient plane are divided into four parts, and each gradient direction falls into one part. Then they implement the interpolation with one-dimension (1-D) cubic convolution interpolation perpendicular to the gradient direction.

We have studied one interpolation algorithm presented in [6]. Here they have applied threshold and Wavelet lifting transform based denoising method first on image and then interpolation algorithm. We have given steps of that algorithm here.

\section{If (value of pixel < threshold) than}

$$
\begin{aligned}
& A(I, j)=A(i-1, j-1)+A(i-1, j+1)+A(i+1, j-1)+A(i+1, j+1) / 4 ; \\
& B(i, j)=B(i-1, j)+B(i+1, j) / 2 ;
\end{aligned}
$$

or

$B(I, j)=B(I, j-1)+B(I, j+1) / 2 ;$

Same way for C,D and E whatever original pixel available, use one of the B's question.

We have refer one algorithm presented in [11], which is the modification in above algorithm. We have given Modified algorithm below.

If (value of pixel < threshold)

than $I(m, n)=(I(m-1, n-1)+I(m-1, n+1)+I(m+1, n-1)+$ $\mathrm{I}(\mathrm{m}+\mathbf{1}, \mathbf{n}+\mathbf{1})) / \mathbf{4}$;

$B=(I(m-1, n)+I(m, n+1)+I(m+1, n)+I(m, n-1)) / 4 ;$

$C=(I(m, n-1)+I(m+1, n)+I(m, n+1)) / 3 ;$

$D=(I(m-1, n)+I(m, n+1)+I(m+1, n)) / 3 ;$

$\mathbf{E}=(\mathbf{I}(\mathbf{m}-\mathbf{1}, \mathbf{n}-\mathbf{1})+\mathbf{I}(\mathbf{m}-\mathbf{1}, \mathbf{n})+\mathbf{I}(\mathbf{m}, \mathbf{n}+\mathbf{1})) / \mathbf{3} ;$

$\mathbf{F}=(\mathbf{I}(\mathbf{m}-\mathbf{1}, \mathbf{n}-\mathbf{1})+\mathbf{I}(\mathbf{m}-\mathbf{1}, \mathbf{n})+\mathbf{I}(\mathbf{m}+\mathbf{1}, \mathbf{n})) / \mathbf{3} ;$

So here more pixel values are considered while calculating interpolated point's value as well as consider the noise present in image too. Here instead of finding mean value of pixels, we can use max or median function of matlab as per the type of image to implement algorithm.

We have studied An Edge-Guided Image Interpolation Algorithm via Directional Filtering and Data Fusion presented in [5]. For edge information they have partition pixels into two directional and orthogonal subsets. Directional interpolation is made for each Subset and two interpolated values are fused. Algorithm presented in [5] work for gray scale images only. Figures below shows how they fill interpolated point.

Modification for this algorithm presented in [12]. Here they made modification so that algorithm works for RGB image also. They have stored each R,G and B components of one image into three different images of two dimension (same as grayscale image) and give that as a input to original algorithm. Finally they have merged all three output arrays into single RGB image[4].

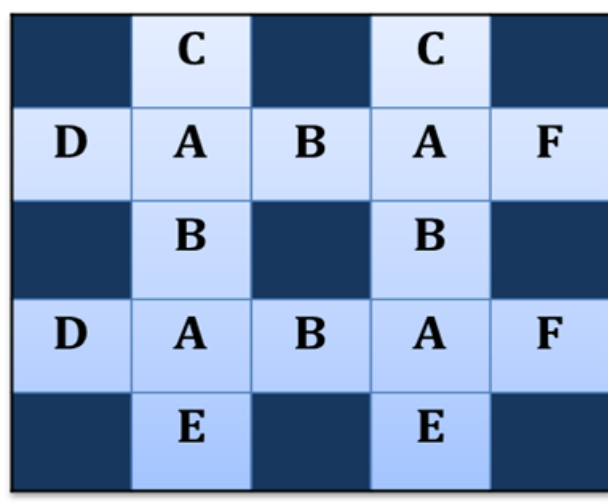

SR Image(2m-1 x 2n-1)

Pixel of Original Image

Figure 3 : Threshold based interpolation[11] 


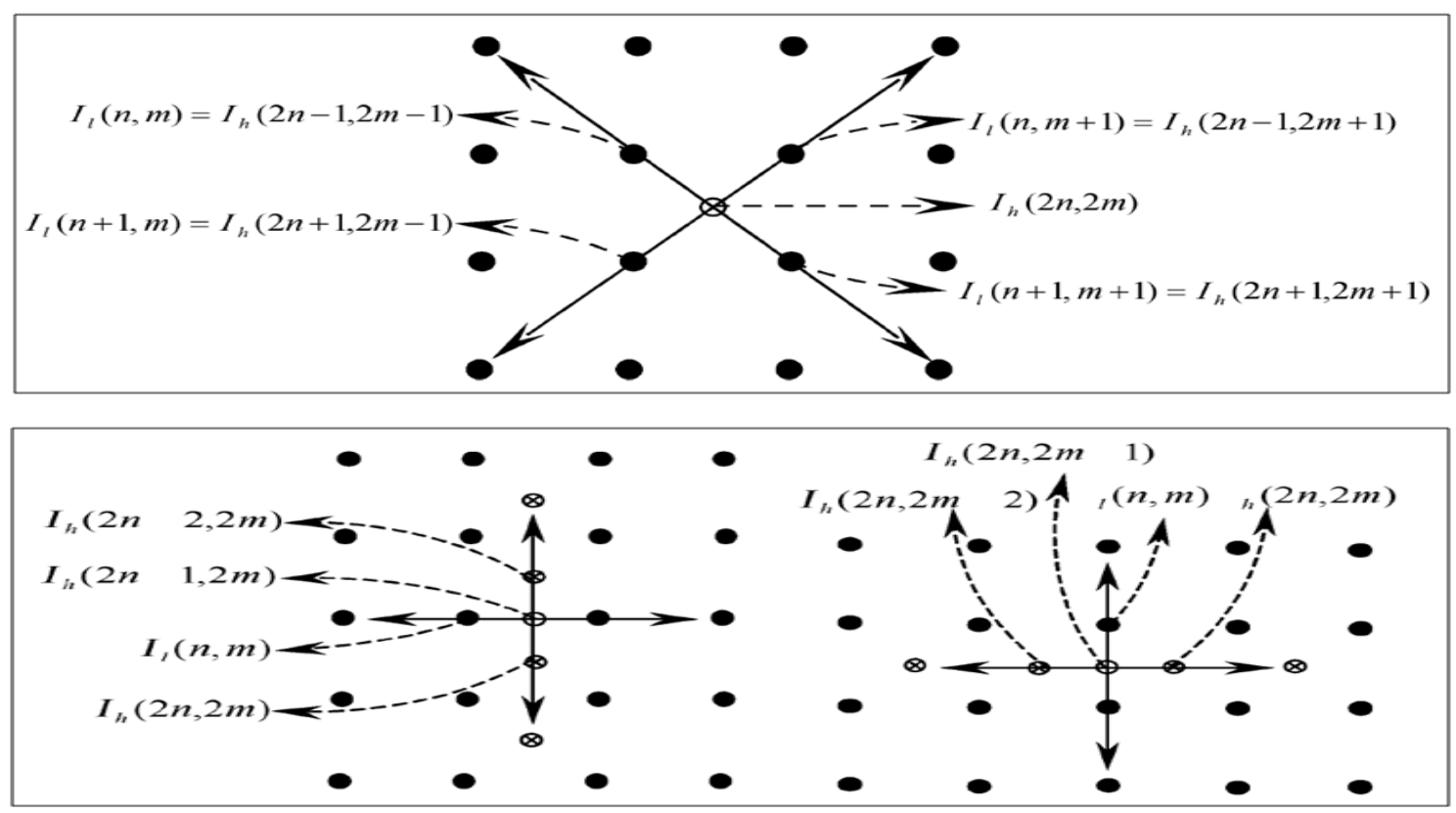

Figure 4 : Edge Guided interpolation[5]

for $\mathrm{i}=1: \mathrm{x}$

$$
\begin{aligned}
& \text { for } j=1: y \\
& \qquad \begin{array}{r}
R(i, j)=\operatorname{Input}(i, j, 1) ; \\
G(i, j)=\operatorname{Input}(i, j, 2) ; \\
B(i, j)=\operatorname{Input}(i, j, 3) ;
\end{array}
\end{aligned}
$$

end

We have studied "image up-sampling using DWT presented in [9]. Here they have used 9/7 bi-orthogonal spline based DWT. Presented algorithm preserve the edges and color of end

for $\mathrm{i}=1:(2 * \mathrm{x})$

for $\mathrm{j}=1$ : $(2 * y)$

$\operatorname{RGB}(\mathrm{i}, \mathrm{j}, 1)=\operatorname{Output}(\mathrm{i}, \mathrm{j})$;

end

end \% same way $\operatorname{RGB}(\mathrm{i}, \mathrm{j}, 2)$ and $\mathrm{RGB}(\mathrm{i}, \mathrm{j}, 3)$ is achieved

original image. Below we have shown the figure, how they do image up-sampling (Here $\mathrm{S}$ is scaling factor and $\mathrm{I}$ is original image).

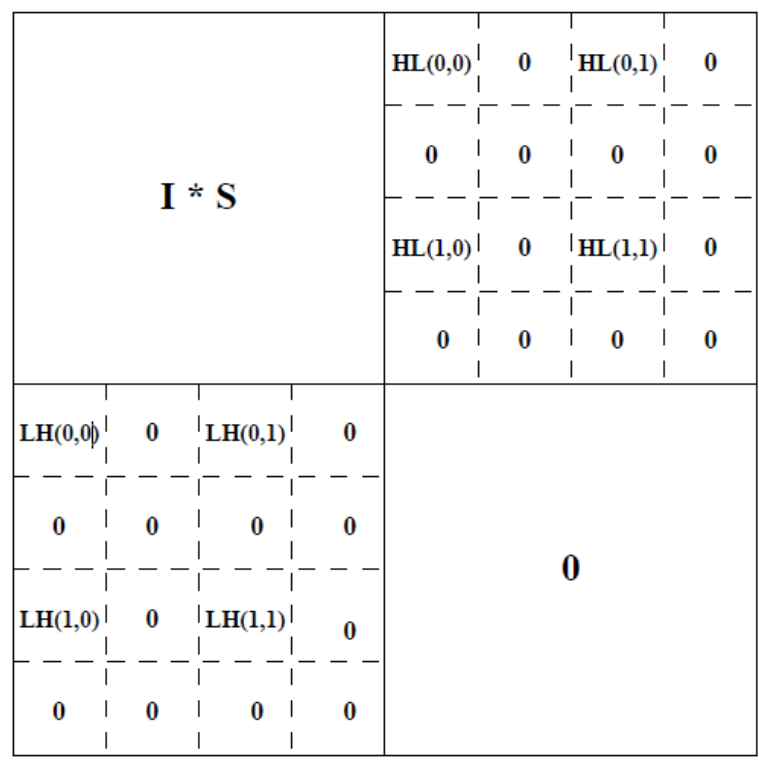

Figure 5 : DWT based up sampling[9]

\section{EXPERIMENT RESULTS AND \\ COMPARISON}

We have used Photoshop [1] for basic methods and for other algorithms, we have implemented basic algorithms in matlab.
All the experiments have been done on the laptop with configuration Intel i3 processor, 4GB RAM and $512 \mathrm{MB}$ graphics card. Experiment we have done on LINA and Monalisa images of the size $256 \times 256$. We have used Pick Signal to Noise Ratio (PSNR) for comparison of all 
algorithms. Visual quality is the most important parameter for the effectiveness of algorithm. Below we have shown all comparison. We have shown the snapshot of Photoshop also in which we can apply basic methods. The PSNR is defined as: PSNR $=20 \cdot \log 10(\mathrm{MAX}$ i $/ \sqrt{\mathrm{MSE}})$. We have compare algorithms in terms of their computation time, complexity of algorithm, PSNR and visual quality.

\section{CONCLUSION AND FUTURE WORK}

In this paper we have shown different interpolation algorithms. We have implemented all algorithms and done the comparison. For commercial tools and applications which are not very important(use for commercial purpose only), nonadaptive algorithms are better to use while in application like medical, bioinformatics, satellite images, adaptive methods are good to use.

In future we would like to modify above algorithms so it will work better as well as we would like to implement our own algorithm for interpolation based on wavelet.

\section{REFERENCES}
[1] Adobe ${ }^{\circledR}$
CS2
software.

(http://www.adobe.com/products/photoshop/)

[2] D. Kirk, Graphics Gems III (IBM Version), Academic Press, San Diego, CA, 1992.

[3] G. Sharma and H.J. Trussell, Digital Color Imaging, IEEE Transactions on Image Processing, 6 (7) (1997) 901-932.

[4] Image Types :: Displaying Bit-Mapped Images : (MATLAB®),http://www.mathworks.in/help/techdoc/cre ating_plots/f2-10709.html

[5] Lei Zhang, Xiaolin Wu, "An Edge-Guided Image Interpolation Algorithm via Directional Filtering and Data Fusion", IEEE TRANSACTIONS ON IMAGE PROCESSING, VOL. 15, NO. 8, AUGUST 2006.

[6] Liyakathunisa \& C.N. Ravi Kumar, "A Novel and Efficient Lifting Scheme based Super Resolution Reconstruction for Early Detection of Cancer in Low Resolution Mammogram Images", International Journal of Biometrics and Bioinformatics (IJBB), Volume (5) : Issue (2) : 2011.

[7] M.D. Fairchild, Color Appearance Models, Reading, MA: Addison Wesley, 1997.
[8] P. Thevenaz, T. Blu and M. Unser, "Image Interpolation and Resampling," Handbook of Medical Imaging, Processing and Analysis, pp. 393-420, 2000.

[9] Ping-Sing Tsai, Tinku Acharya," Image Up-Sampling Using Discrete Wavelet Transform”, 9th JCIS 2006, 8 to 11 October 2006

[10] Recommendations on Uniform Color Spaces, Color Difference Equations, Psychometric Color Terms, C.I.E, Supplement no. 2 to CIE publication no. 15(E.-1 31) 1971/(TC-1.3), 1978

[11] sapan Naik, Viral Borisagar, " Super-Resolution using Interpolation and Wavelet lifting scheme" , IJCA journals, VOL 40, 2012.

[12] Sapan Naik, Viral Borisagar," A novel Super Resolution Algorithm using Interpolation and LWT based denoising method ", IJIP journal, VOL. 6, issue 4,2012.

[13] T. Acharya and A. K. Ray, Image Processing: Principles and Applications, John Wiley \& Sons, Inc., Hoboken, NJ, 2005.

[14] T. Lehmann, C. Gonner, and K. Spetzer, "Survey: Interpolation Methods in Medical Image Processing," IEEE Trans. Med. Imaging, 18:1049-1067,1999.

[15] www.mathwork.com

[16] www.wikipedia.com

[17] Xiao-feng Wang, He-fei Ling, "An Edge-Adaptive Interpolation Algorithm for Super-Resolution Reconstruction", 2010 International Conference on Multimedia Information Networking and Security.

[18] Chunmei Liu, Lei Xu, "A New Image Interpolation Algorithm Based on Pulse-Coupled Neural Networks", 2011 International Conference on Computational and Information Sciences.

[19] Sanjay Kumar Maurya, Pavan Kumar Mishra, "Image Enhancement by Intensity Based Interpolation and Selective Threshold", 2012 International Conference on Communication Systems and Network Technologies.

[20] Bing Yang, Zhiyong Gao, Xiaoyun Zhang, "Principal Components Analysis-Based Edge-Directed Image Interpolation”, 2012 IEEE International Conference on Multimedia and Expo (ICME).

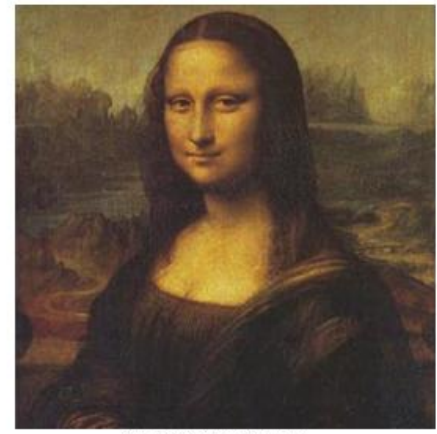

MONALISA

Figure 6 : Original Input Images 


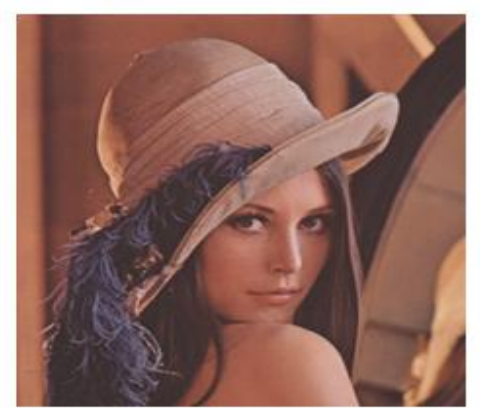

a

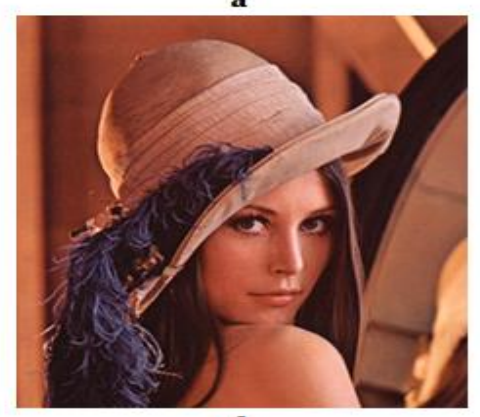

d

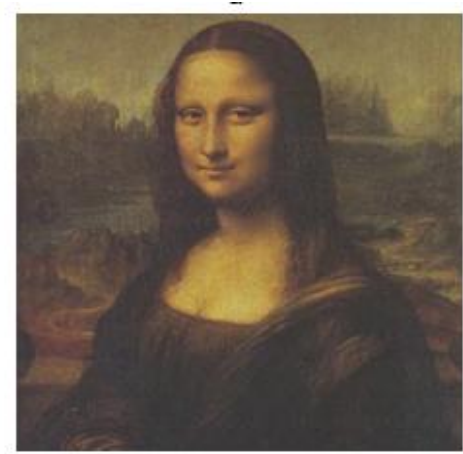

a

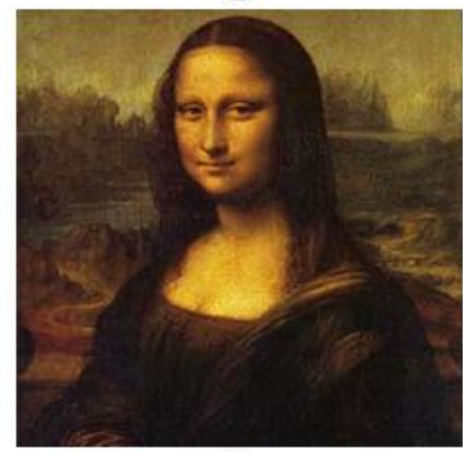

d

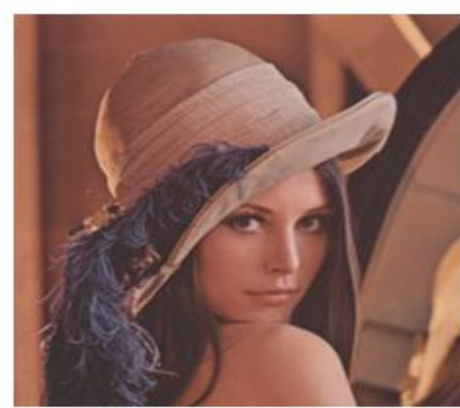

b

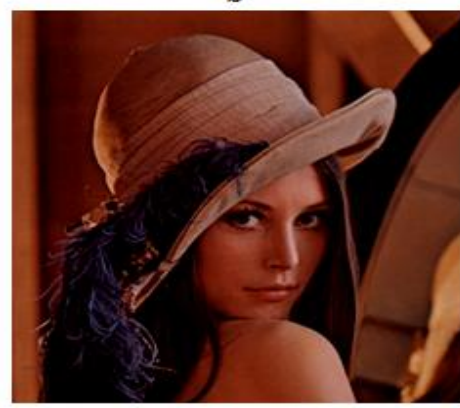

e

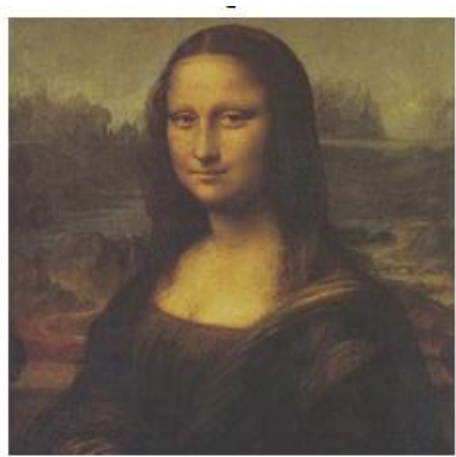

b

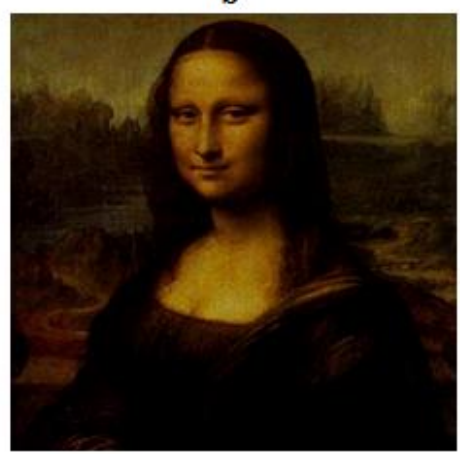

e

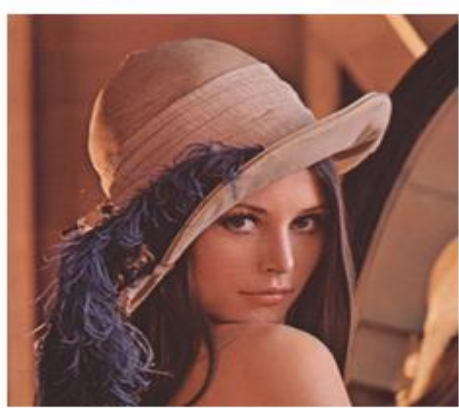

c

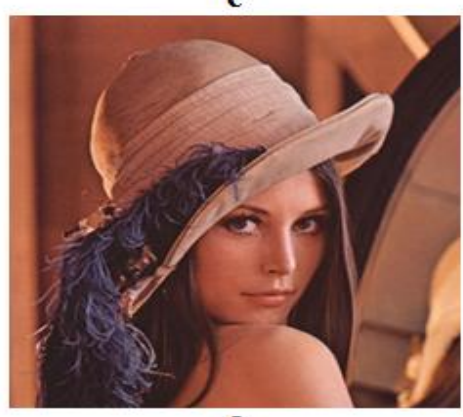

f

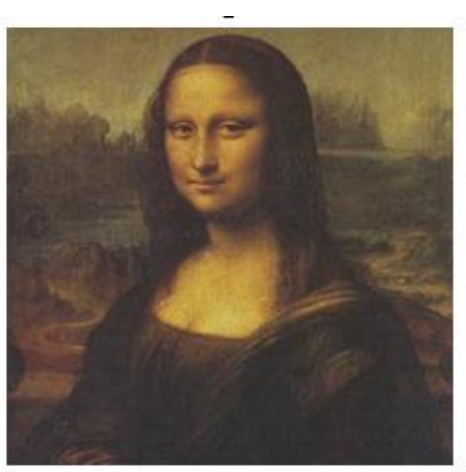

c

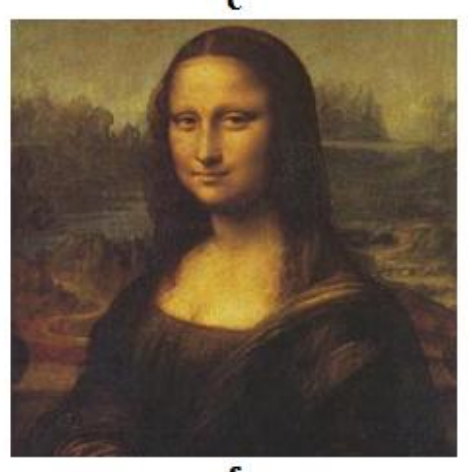

f

Figure 7 : LENA image, a. nearest neighbour, b. Bilinear, c. Bicubic, d. Edge Guided, e. DWT based, f. Threshhold based adeptive[1,15] 
Table 2 : Comparison of different interpolation algorithms

\begin{tabular}{|c|c|c|c|}
\hline Interpolation Algorithms & \multicolumn{2}{|c|}{$\begin{array}{c}\text { PSNR(dB) } \\
\text { LENA }\end{array}$} \\
\hline MONALISA
\end{tabular}

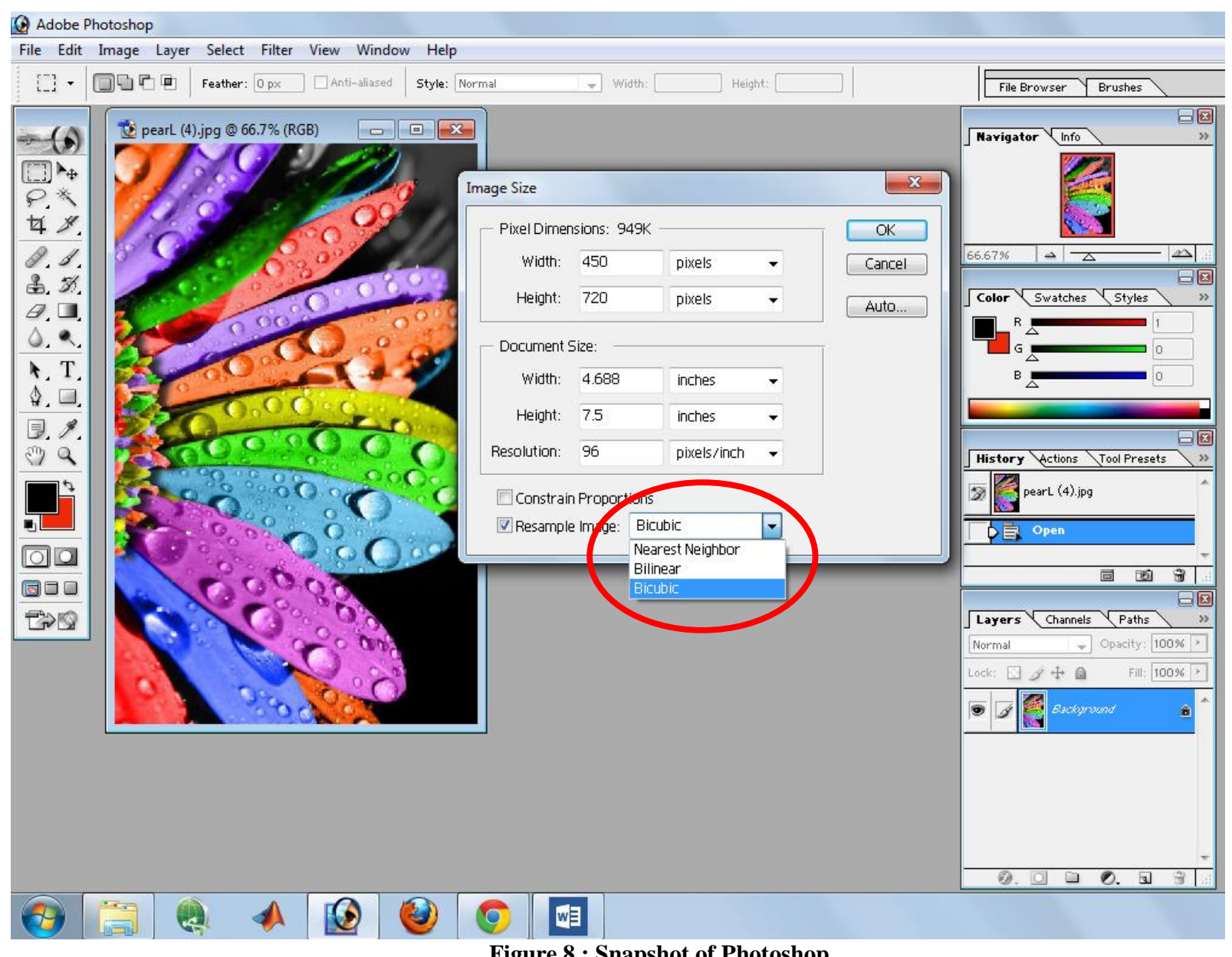

Figure 8 : Snapshot of Photoshop 